\title{
Non-Equilibrium High Speed Air Flow over Spherical Blunt Bodies
}

\author{
Sergey Alexandrov ${ }^{1}$, Alexander Zdor ${ }^{1}$, Alexander Kireev ${ }^{1}$, \\ Sergey Chernov ${ }^{1}$, Vladimir Yumashev ${ }^{1}$ \\ ${ }^{1}$ Central Aerohydrodynamic Institute (TsAGI), Zhukovsky, Moscow Region, 140181, Russia \\ a_kireev1950@mail.ru, yumashev@mail.ru
}

\begin{abstract}
Based on two-dimensional full Navier-Stokes equations the flow about stagnation point of spherically blunted body at hypersonic flight in the atmosphere of the Earth is investigated. The problem is being solved taking into account non-equilibrium physical-chemical processes (vibration relaxation, vibration-dissociation coupling, dissociation, chemical reactions, ionization). Convective heat fluxes at the stagnation point are compared to ones obtained using simplified engineering technique.
\end{abstract}

Keywords: non-equilibrium high temperature air, heat flux, numerical simulation, physicalchemical processes
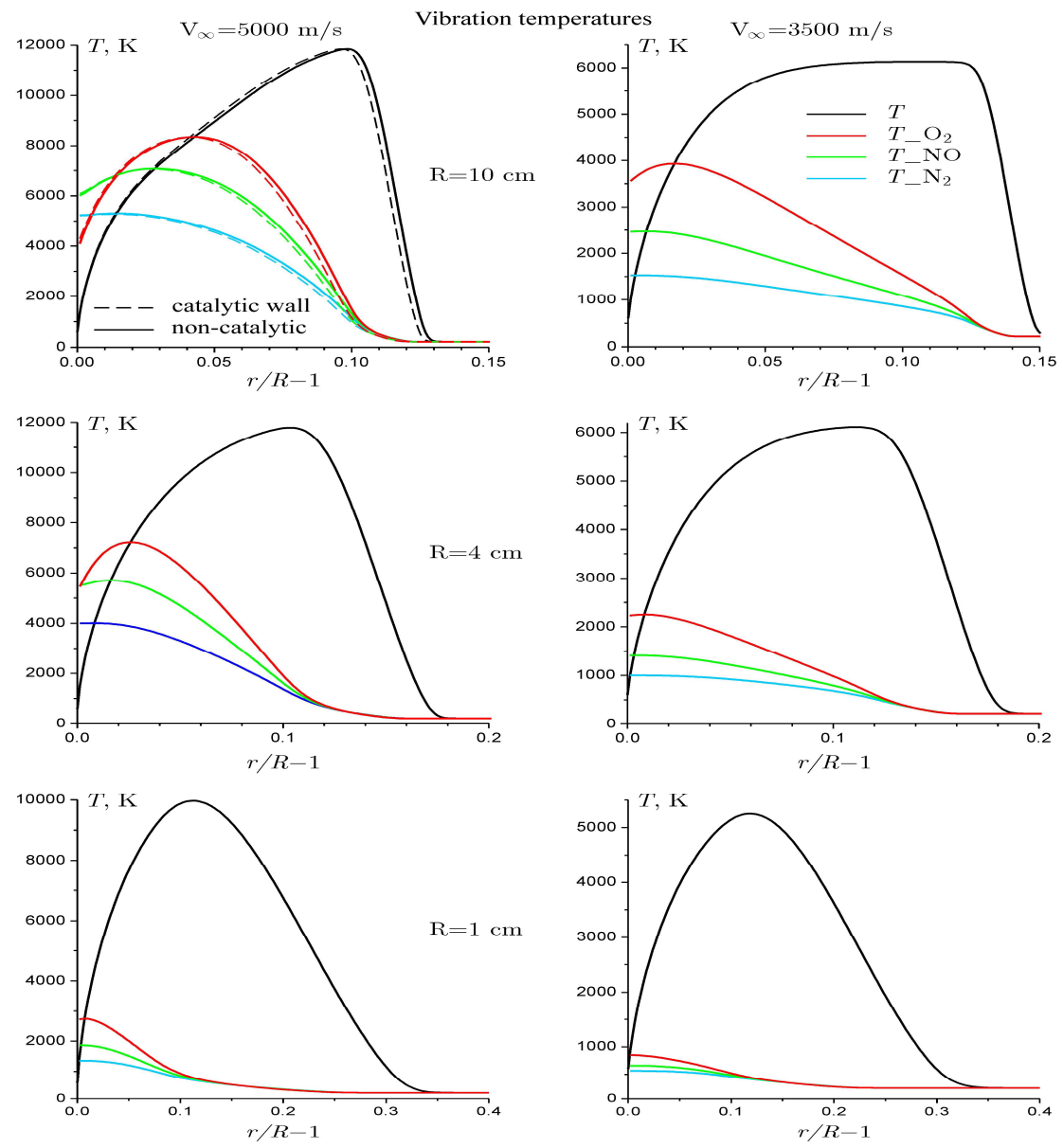

Vibrational temperatures of diatomic molecules and translational temperature along stagnation line at different bluntness radiuses. Left column $V_{\infty}=5000 \mathrm{~m} / \mathrm{s}$, right column $V_{\infty}=3500 \mathrm{~m} / \mathrm{s}, H=70 \mathrm{~km}$. 


\title{
Особенности неравновесного гиперзвукового течения воздуха в окрестности сферического затупления
}

\author{
С.В. Александров ${ }^{1}$, А.Г. Здор ${ }^{1}$, А.Ю. Киреев ${ }^{1}$, С.В. Чернов ${ }^{1}$, В.Л. Юмашев ${ }^{1}$ \\ ${ }^{1}$ Центральный аэрогидродинамический институт им. проф. Н.Е. Жуковского (ЦАГИ), \\ Россия, г. Жуковский, Московская обл., 140181 \\ a_kireev1950@mail.ru, yumashev@mail.ru
}

\begin{abstract}
Аннотация
На основе полных уравнений Навье-Стокса исследуется течение в окрестности точки торможения при гиперзвуковом движении сферически затупленного аппарата в атмосфере Земли. Задача решается с учетом неравновесного протекания физико- химических процессов (колебательная релаксация, колебательно- диссоциационное взаимодействие, диссоциация, химические реакции, ионизация) в двумерной постановке с помощью разработанного в ЦАГИ программного комплекса HSFlow (High Speed Flow).
\end{abstract}

Ключевые слова: высокоэнтальпийное неравновесное течение, численное моделирование.

\section{1. Введение}

Одной из наиболее важных задач при проектировании космических и гиперзвуковых аппаратов является определение тепловых потоков к их поверхности при полете в атмосфере с большими сверхзвуковыми скоростями. В условиях экстремально высоких температур в ударном слое около тела важное значение имеет учет неравновесного протекания физикохимических процессов. Исследованию проблемы численного моделированию вязких, неравновесных течений около затупленных тел гиперзвуковым потоком воздуха и газовых смесей различного состава на основе полных уравнений Навье-Стокса посвящено большое количество работ и разработаны ряд значимых программных комплексов. Поскольку работа не является обзором, приведем лишь некоторые из них, которые считаем наиболее интересными. Уравнения сохранения, кинетика и тестовые расчеты даются в [1]. Из российских работ необходимо упомянуть работы [2-3], где приводятся методика расчета и результаты применения разработанного их авторами программного комплекса описания неравновесновесных 2-D и 3-D течений при полете аппаратов различного типа.

Учитывая важность задач неравновесных течений в практических приложениях, в данной работе сделана попытка применения программного комплекса HSFlow в 2-D постановке для расчета обтекания тела со сферическим затуплением неравновесным гиперзвуковым потоком воздуха.

Здесь излагаются методики расчета, а также, инженерные оценки конвективных тепловых потоков при гиперзвуковом движении сферически затупленного аппарата в атмосфере Земли при вариации скорости и радиуса затупления тела. Задача решается с учетом неравновесного протекания физико- химических процессов (колебательная релаксация, колебательно- диссоциационное взаимодействие, диссоциация, химические реакции, ионизация) в двумерной постановке. Реализованы два подхода: 1) на основе полных уравнений Навье-Стокса; 2) инженерный подход для быстрой оценки уровня конвективных тепловых 
потоков к критической точке затупления с учетом разреженности набегающего воздушного потока.

\section{2. Физическая постановка и математическая модель}

Рассматривается вязкое ламинарное течение многокомпонентного газа, в котором происходят неравновесные химические реакции и неравновесное возбуждение колебательных степеней свободы. Течение описывается системой уравнений Навье-Стокса, имеющих вид в векторной форме в декартовых координатах

$$
\frac{\partial \mathbf{A}}{\partial t}+\frac{\partial \mathbf{B}}{\partial x}+\frac{\partial \mathbf{C}}{\partial y}+\frac{\partial \mathbf{D}}{\partial z}=\mathbf{R}
$$

Векторы $\mathbf{A}, \mathbf{B}, \mathbf{C}, \mathbf{D}, \mathbf{W}$ представляют собой

$$
\begin{gathered}
\mathbf{A}=\left[\begin{array}{c}
\rho \\
\rho u \\
\rho v \\
\rho w \\
\rho E \\
\rho c_{i}
\end{array}\right], \mathbf{B}=\left[\begin{array}{c}
\rho u \\
\rho u^{2}+p+\tau_{x x} \\
\rho u v+\tau_{x y} \\
\rho u w+\tau_{x z} \\
\rho u H+q_{x} \\
\rho u c_{i}+d_{x}^{i}
\end{array}\right], \mathbf{C}=\left[\begin{array}{c}
\rho v \\
\rho u v+\tau_{y x} \\
\rho v^{2}+p+\tau_{y y} \\
\rho v w+\tau_{y z} \\
\rho v H+q_{y} \\
\rho v c_{i}+d_{y}^{i}
\end{array}\right], \mathbf{D}=\left[\begin{array}{c}
\rho w \\
\rho u w+\tau_{z x} \\
\rho v w+\tau_{z y} \\
\rho w^{2}+p+\tau_{z z} \\
\rho w H+q_{z} \\
\rho w c_{i}+d_{z}^{i}
\end{array}\right], \mathbf{R}=\left[\begin{array}{c}
0 \\
0 \\
0 \\
0 \\
0 \\
r_{i}
\end{array}\right], \\
i=1, \ldots, N-1 .
\end{gathered}
$$

Здесь $u, v, w$ - декартовы компоненты вектора скорости; $p$ - давление; $\rho$ - плотность; $E$ полная энергия в единице массы; $H$ - полная энтальпия; $\tau$ - тензор вязких напряжений; $\mathbf{q}$ - вектор потоков энергии за счет молекулярного переноса; $c_{i}$ - массовые доли компонент смеси; $\mathbf{d}^{i}$ - векторы диффузионных потоков компонент; $r_{i}$ - скорости производства компонент в единице объема; $i$ - номера компонент; $N$ - суммарное число компонент.

Тензор вязких напряжений связан линейно с тензором скоростей деформаций

$$
\tau=-\mu \mathbf{S},
$$

где $\mu$ - динамическая вязкость, а тензор скоростей деформаций имеет компоненты

$$
\begin{array}{ccc}
s_{x x}=2 \frac{\partial u}{\partial x}-\frac{2}{3} \operatorname{div} \mathbf{V}, & s_{y y}=2 \frac{\partial v}{\partial y}-\frac{2}{3} \operatorname{div} \mathbf{V}, & s_{z z}=2 \frac{\partial w}{\partial z}-\frac{2}{3} \operatorname{div} \mathbf{V}, \\
s_{x y}=s_{y x}=\frac{\partial u}{\partial y}+\frac{\partial v}{\partial x}, & s_{x z}=s_{z x}=\frac{\partial u}{\partial z}+\frac{\partial w}{\partial x}, & s_{y z}=s_{z y}=\frac{\partial v}{\partial z}+\frac{\partial w}{\partial y}
\end{array}
$$

Вектор q складывается из действий вязкости, теплопроводности и диффузии, векторы диффузионных потоков $\mathbf{d}^{i}$ даются законом Фика

$$
\mathbf{q}=-\lambda \nabla T+\tau \mathbf{V}+\sum_{i=1}^{N} h_{i} \mathbf{d}^{i}, \quad \mathbf{d}^{i}=-\rho D_{i} \nabla c_{i} .
$$

Используется модель бинарной диффузии, поскольку преобладающей компонентой является молекулярный азот, по сравнению с которым прочие компоненты можно считать малыми примесями. Коэффициент вязкости $\mu$ выражается степенной функцией температуры с показателем $\omega=0.731$, коэффициенты теплопроводности $\lambda$ и диффузии $D_{i}$ следуют из чисел Прандтля и Шмидта 


$$
\frac{\mu}{\mu_{\infty}}=\left(\frac{T}{T_{\infty}}\right)^{\omega}, \quad \operatorname{Pr}=\frac{\mu C_{p}}{\lambda}=0.7, \quad \mathrm{Sc}_{i}=\frac{\mu}{\rho D_{i}}=0.5
$$

Параметры течения связаны термодинамическими зависимостями

$$
p=\rho T \sum_{i=1}^{N} c_{i} R_{i}, \quad H=\sum_{i=1}^{N} c_{i} h_{i}+\frac{u^{2}+v^{2}+w^{2}}{2}, \quad h_{i}=h_{0 i}+R_{i} \sum_{j=1}^{L} v_{i j} E_{v j}+C_{p i} T
$$

Здесь $T$ - температура; $h_{i}$ - энтальпии компонент; $h_{0 i}-$ их энтальпии образования; $R_{i}$ и $C_{p i}$ - газовые постоянные и теплоемкости компонент (без учета колебательной энергии); $E_{v j}$ - колебательные энергии компонент, измеряемые в Кельвинах; $j$ - номера колебательных мод; $L$ - их суммарное число; $v_{i j}$ - коэффициенты кратности колебательных мод (для каждого $j$ существует единственное $i$, при котором $\left.v_{i j} \neq 0\right)$. Колебательные энергии компонент $E_{v j}$ измеряются в градусах Кельвина. Включение энтальпии образования компонент в полную энергию позволяет обойтись без источникового члена в уравнении энергии. Энергия колебательных степеней свободы подчиняется закону Ландау-Теллера

$$
\frac{\mathrm{d} E_{v j}}{\mathrm{~d} t}=\frac{E_{v j}^{0}-E_{v j}}{\tau_{v j}}, \quad j=1, \ldots, L
$$

Чтобы включить эти уравнения в общую систему, следует привести их к виду, аналогичному законам сохранения массы, импульса и энергии:

$$
\frac{\partial \rho E_{v j}}{\partial t}+\frac{\partial \rho u E_{v j}}{\partial x}+\frac{\partial \rho v E_{v j}}{\partial y}+\frac{\partial \rho w E_{v j}}{\partial z}=\rho \frac{E_{v j}^{0}-E_{v j}}{\tau_{v j}}, \quad j=1, \ldots, L
$$

\section{3. Модель физико-химических процессов и численное решение}

Система химических реакций может быть представлена в общем виде, чтобы процедура решения не была привязана к частным случаям. Предполагается, что между $N$ компонентами происходят $K$ реакций, причем реакция с номером $j$ описывается обобщенной формулой

$$
\alpha_{j 1} Z_{1}+\alpha_{j 2} Z_{2}+\cdots+\alpha_{j N} Z_{N}=\beta_{j 1} Z_{1}+\beta_{j 2} Z_{2}+\cdots+\beta_{j N} Z_{N}
$$

Здесь $\alpha_{j i}$ и $\beta_{j i}$ - стехиометрические коэффициенты; $Z_{i}-$ химические наименования компонент. С каждой стороны формулы редко бывает более трех членов, поэтому в матрицах $\alpha_{j i}$ и $\beta_{j i}$ большинство элементов - нули. Скорость производства $i$-й компоненты определяется как

$$
\begin{gathered}
r_{i}=m_{i} \sum_{j=1}^{K}\left(\beta_{j i}-\alpha_{j i}\right)\left(G_{f j}-G_{r j}\right), \\
G_{f j}=A_{f j} T^{B_{f j}} e^{-\frac{D_{f j}}{T}} \prod_{i=1}^{N}\left(\frac{\rho c_{i}}{m_{i}}\right)^{\alpha_{j i}}, \quad G_{r j}=A_{r j} T^{B_{r j}} e^{-\frac{D_{r j}}{T}} \prod_{i=1}^{N}\left(\frac{\rho c_{i}}{m_{i}}\right)^{\beta_{j i}}
\end{gathered}
$$

Здесь $m_{i}$ - молекулярные веса компонент; $G_{f j}$ и $G_{r j}$ - скорости прямых и обратных реакций; $A_{f j}, B_{f j}, E_{f j}, A_{r j}, B_{r j}, E_{r j}$ - параметры закона Аррениуса. В данном случае рассматривается модель воздуха из десяти компонент (табл. 1) при наличии девяти реакций между ними (табл. 2). Параметры реакций соответствуют [6], при этом учитываются коэффициенты эффективности третьей частицы в реакциях диссоциации-рекомбинации (табл. 3). 
Таблийа 1

Параметры компонент газовой смеси

\begin{tabular}{|c|c|c|c|c|c|c|}
\hline Компонента & $m$ & $\begin{array}{c}\text { Степени } \\
\text { свободы }\end{array}$ & $h_{0} / R, \mathrm{~K}$ & $\theta, \mathrm{K}$ & $\sigma, \mathrm{m}^{2}$ & $E_{d}, \mathrm{~K}$ \\
\hline $\mathrm{O}_{2}$ & 32 & 5 & 0 & 2256 & $3.0 \times 10^{-21}$ & 29875 \\
\hline $\mathrm{O}$ & 16 & 3 & 29760 & - & - & - \\
\hline $\mathrm{N}$ & 14 & 3 & 56600 & - & - & - \\
\hline $\mathrm{NO}$ & 30 & 5 & 10810 & 2710 & $3.0 \times 10^{-21}$ & 37750 \\
\hline $\mathrm{N}_{2}$ & 28 & 5 & 0 & 3354 & $3.0 \times 10^{-21}$ & 56600 \\
\hline $\mathrm{O}^{+}$ & 16 & 3 & 153338 & - & - & - \\
\hline $\mathrm{O}_{2}^{+}$ & 32 & 5 & 140000 & 2256 & - & 2256 \\
\hline $\mathrm{NO}^{+}$ & 30 & 5 & 126000 & 2714 & - & 2714 \\
\hline $\mathrm{N}_{2}^{+}$ & 28 & 5 & 181000 & 3354 & - & 3354 \\
\hline $\mathrm{e}^{-}$ & 0.00055 & 3 & 0 & - & - & - \\
\hline
\end{tabular}

Таблииа 2

Параметры химических реакций

\begin{tabular}{|c|c|c|c|c|c|c|c|}
\hline Реакция & $A_{f}$ & $B_{f}$ & $D_{f}$ & $A_{r}$ & $B_{r}$ & $D_{r}$ & $E_{d}$ \\
\hline $\mathrm{N}_{2}+\mathrm{M}=\mathrm{N}+\mathrm{N}+\mathrm{M}$ & $7.0 \times 10^{18}$ & -1.6 & 113200 & $1.015 \times 10^{13}$ & -1.24 & 0 & 56600 \\
\hline $\mathrm{O}_{2}+\mathrm{M}=\mathrm{O}+\mathrm{O}+\mathrm{M}$ & $2.0 \times 10^{18}$ & -1.5 & 59750 & $1.171 \times 10^{13}$ & -1.19 & 0 & 29875 \\
\hline $\mathrm{NO}+\mathrm{M}=\mathrm{N}+\mathrm{O}+\mathrm{M}$ & $5.0 \times 10^{12}$ & 0.0 & 75500 & $1.129 \times 10^{8}$ & 0.27 & 0 & 37750 \\
\hline $\mathrm{O}+\mathrm{NO}=\mathrm{N}+\mathrm{O}_{2}$ & $2.8 \times 10^{6}$ & 1.0 & 20000 & $1.1 \times 10^{7}$ & 1.0 & 4000 & - \\
\hline $\mathrm{O}+\mathrm{N}_{2}=\mathrm{N}+\mathrm{NO}$ & $2.04 \times 10^{9}$ & 0.5 & 38000 & $4.4 \times 10^{8}$ & 0.5 & 0 & - \\
\hline $\mathrm{N}+\mathrm{O}=\mathrm{NO}^{+}+\mathrm{e}^{-}$ & $2.56 \times 10^{9}$ & 0.0 & 32200 & $6.7 \times 10^{18}$ & -1.5 & 0 & - \\
\hline $\mathrm{O}+\mathrm{O}=\mathrm{O}_{2}^{+}+\mathrm{e}^{-}$ & 0.71 & 2.7 & 80600 & $1.492 \times 10^{14}$ & -0.06 & 0 & - \\
\hline $\mathrm{N}+\mathrm{N}=\mathrm{N}_{2}^{+}+\mathrm{e}^{-}$ & $4.44 \times 10^{7}$ & 0.7 & 67500 & $1.5 \times 10^{19}$ & -1.5 & 0 & - \\
\hline $\mathrm{O}+\mathrm{e}^{-}=\mathrm{O}^{+}+\mathrm{e}^{-}+\mathrm{e}^{-}$ & $3.9 \times 10^{30}$ & -3.78 & 158500 & $3.686 \times 10^{38}$ & -5.89 & 0 & - \\
\hline
\end{tabular}

Таблица 3

Коэффициенты эффективности третьей частицы в реакциях диссоциации-рекомбинации

\begin{tabular}{|c|c|c|c|c|c|c|c|c|c|c|}
\hline Тип 3-й частицы & $\mathrm{N}$ & $\mathrm{O}$ & $\mathrm{NO}$ & $\mathrm{N}_{2}$ & $\mathrm{O}_{2}$ & $\mathrm{O}^{+}$ & $\mathrm{O}_{2}^{+}$ & $\mathrm{NO}^{+}$ & $\mathrm{N}_{2}^{+}$ & $\mathrm{e}^{-}$ \\
\hline $\mathrm{M}\left(\mathrm{N}_{2}\right)$ & 4.3 & 4.3 & 1.0 & 1.0 & 1.0 & 4.3 & 1.0 & 1.0 & 1.0 & 1.0 \\
\hline $\mathrm{M}\left(\mathrm{O}_{2}\right)$ & 5.0 & 5.0 & 1.0 & 1.0 & 1.0 & 5.0 & 1.0 & 1.0 & 1.0 & 1.0 \\
\hline $\mathrm{M}(\mathrm{NO})$ & 22.0 & 22.0 & 22.0 & 1.0 & 1.0 & 22.0 & 1.0 & 22.0 & 1.0 & 1.0 \\
\hline
\end{tabular}

Равновесные значения колебательной энергии определяются законом Дебая

$$
E_{v j}^{0}=\frac{\theta_{j}}{e^{\theta_{j} / T}-1}
$$

где $\theta_{j}$ - дебаевские температуры колебательных мод. Характерное время колебательной релаксации определяется по формуле Милликена-Уайта [4] с введением поправки Парка [5] 


$$
\begin{gathered}
\tau_{v j}=\left(\sum_{i=1}^{K} \frac{c_{i}}{\tau_{i j}}\right)^{-1}+\tau_{p}, \quad \tau_{i j}=\frac{9.8 \times 10^{-4}}{p} e^{a_{i j}\left(T^{-1 / 3}-b_{i j}\right)}, \\
a_{i j}=0.00116 m_{i j}^{1 / 2} \theta_{j}^{4 / 3}, \quad b_{i j}=0.015 m_{i j}^{1 / 4}, \quad m_{i j}=\frac{m_{i} m_{j}}{m_{i}+m_{j}}, \\
\tau_{p}=\frac{1}{\rho N_{A} \sigma} \sqrt{\frac{\pi}{8 R_{0} T}\left(\sum_{i=1}^{K} \frac{c_{i}}{m_{i}}\right)^{-3 / 2}\left(\frac{T}{50000}\right)^{2}}
\end{gathered}
$$

Здесь $N_{A}$ - число Авогадро; $\sigma$ - эффективное сечение столкновения молекул; $R_{0}-$ универсальная газовая постоянная. Значения параметров $\theta$ и $\sigma$ для рассматриваемых компонент приведены в табл. 1.

Известно, что неравновесное возбуждение колебательных степеней свободы влияет на процесс диссоциации. Колебательно-диссоциационное взаимодействие учитывается путем умножения скорости реакции диссоциации на поправочный множитель [6]

$$
\frac{E_{v}^{0}+\theta}{E_{v}+\theta} e^{E_{d}\left(1 / T-1 / T_{v}\right)}
$$

Значения параметра $E_{d}$, связанного с энергией диссоциации, приведены в табл. 2 для соответствующих реакций. Колебательная температура $T_{v}$ по определению находится из соотношения

$$
E_{v}=\frac{\theta}{e^{\theta / T_{v}}-1}, \quad T_{v}=\frac{\theta}{\ln \left(1+\theta / E_{v}\right)}
$$

Решение поставленной задачи выполняется при помощи разработанной в ЦАГИ программы HSflow [7], реализующей изложенную математическую модель. Программа позволяет рассчитывать двумерные и трехмерные течения вязкого газа на многоблочной регулярной сетке. Поскольку расчетная сетка чаще всего бывает неравномерной и криволинейной, выполняется переход к обобщенным координатам $\xi, \eta, \varsigma$, в которых сетка является равномерной и прямоугольной. На практике в качестве обобщенных координат рассматриваются просто номера узлов сетки по трем сеточным измерениям. Поскольку декартовы координаты узлов заведомо известны, это сразу дает связи

$$
x=x(\xi, \eta, \zeta), \quad y=y(\xi, \eta, \zeta), \quad z=z(\xi, \eta, \zeta)
$$

В обобщенных координатах основная система уравнений имеет вид

$$
\frac{\partial \mathbf{E}}{\partial t}+\frac{\partial \mathbf{F}}{\partial \xi}+\frac{\partial \mathbf{G}}{\partial \eta}+\frac{\partial \mathbf{H}}{\partial \zeta}=\mathbf{W}
$$

Между векторами в обобщенной и декартовой системах координат действуют связи

$$
\begin{gathered}
\mathbf{E}=J \mathbf{A}, \quad \mathbf{F}=J\left(\mathbf{B} \frac{\partial \xi}{\partial x}+\mathbf{C} \frac{\partial \xi}{\partial y}+\mathbf{D} \frac{\partial \xi}{\partial z}\right), \quad \mathbf{G}=J\left(\mathbf{B} \frac{\partial \eta}{\partial x}+\mathbf{C} \frac{\partial \eta}{\partial y}+\mathbf{D} \frac{\partial \eta}{\partial z}\right), \\
\mathbf{H}=J\left(\mathbf{B} \frac{\partial \zeta}{\partial x}+\mathbf{C} \frac{\partial \zeta}{\partial y}+\mathbf{D} \frac{\partial \zeta}{\partial z}\right), \quad \mathbf{W}=J \mathbf{R}, \quad J=\frac{\partial(x, y, z)}{\partial(\xi, \eta, \zeta)}
\end{gathered}
$$


В таком виде система уравнений аппроксимируется в узлах сетки конечными разностями так, что выполняются разностные аналоги законов сохранения массы, импульса и энергии в условных контрольных объемах, окружающих каждый узел. Для вычисления потоков массы, импульса и энергии между контрольными объемами используются современные варианты метода Годунова, в основе которого лежит концепция распада произвольного разрыва газодинамических параметров (задача Римана) [8-10]. Метод Годунова обеспечивает только первый порядок аппроксимации, которого недостаточно для расчета вязких течений из-за сопутствующей ему схемной вязкости. Повышение порядка аппроксимации до второго достигается применением метода Колгана и его развития [11-13], использующих принцип минимума производных при определении градиентов искомых функций.

В результате разностной аппроксимации возникает очень большая система алгебраических уравнений, которую условно можно представить в векторном виде

$$
\mathbf{P}(\mathbf{X})=0
$$

Вектор $\mathbf{P}$ содержит множество уравнений, вектор $\mathbf{X}$ - множество искомых величин во всех узлах расчетной сетки. Длина каждого из векторов равна суммарному числу узлов, умноженному на число неизвестных в каждом узле, и может доходить до $10^{7} \div 10^{9}$. Ввиду нелинейности система решается итерационным методом Ньютона-Рафсона, на каждом шаге которого возникает линейная система того же размера

$$
\frac{D \mathbf{P}\left(\mathbf{X}^{(k)}\right)}{D \mathbf{X}}\left(\mathbf{X}^{(k+1)}-\mathbf{X}^{(k)}\right)+\beta^{(k)} \mathbf{P}\left(\mathbf{X}^{(k)}\right)=0
$$

Здесь $k$ - номер итерации; $D \mathbf{P} / D \mathbf{X}$ - матрица Якоби; $\beta$ - демпфирующий множитель для улучшения сходимости итераций. Следуя [14], используется автоматическая подстройка $\beta$ под то, как развивается итерационный процесс

$$
\beta^{(k+1)}=-\beta^{(k)} \frac{\left(\Delta^{(k+1)}-\Delta^{(k)}, \Delta^{(k)}\right)}{\left|\Delta^{(k+1)}-\Delta^{(k)}\right|^{2}}, \quad \Delta^{(k)}=\mathbf{X}^{(k)}-\mathbf{X}^{(k-1)}
$$

В целях общности и во избежание громоздких выкладок матрица Якоби вычисляется путем численного дифференцирования. Поскольку в каждом разностном уравнении задействованы только ближайшие узлы, матрица Якоби сильно разрежена и имеет мультидиагональную блочную структуру.

Для решения линейных систем существуют прямые методы типа LU- и QU-разложений. Однако их трудоемкость возрастает пропорционально $n^{3}$, где $n-$ размер системы, поэтому их нереально применять, если $n$ существенно превышает $10^{2} \div 10^{3}$. В таких случаях более эффективными становятся итерационные методы решения линейных систем. В программе HSFlow для этих целей используется обобщенный метод минимальных невязок GMRes [15]. Надежность и скорость сходимости метода подкрепляется применением предобуславливателя [16].

\section{4. Результаты расчетов}

В качестве объекта для исследования выбраны сферические носовые затупления радиусом $1 \mathrm{~cm}, 4$ см и $10 \mathrm{~cm}$, обтекаемые потоком воздуха со скоростью от $3500 \mathrm{~m} / \mathrm{c}$ и $5000 \mathrm{M} / \mathrm{c}$ при параметрах атмосферы на высоте 70 км. На поверхности заданы условия прилипания, температура поверхности принята постоянной, равной $600 \mathrm{~K}$. Рассматриваются предельные случаи полностью каталитической и полностью некаталитической поверхности.

Общий характер поля течения показан на рис. 1 в виде уровней давления и температуры для скорости набегающего потока $5000 \mathrm{~m} / \mathrm{c}$ и трех разных радиусов затупления. При переходе от $R=10$ см к 1 см относительная толщина ударного слоя увеличивается в 2.5 раза. 
Головная ударная волна при $R=4$ см становится более размытой, а при $R=1$ см исчезает и заменяется непрерывным распределением параметров поперек ударного слоя. Температурный максимум в ударном слое при $R=10$ см и 4 см практически одинаковый, а при $R=1 \mathrm{~cm}$ становится заметно ниже.
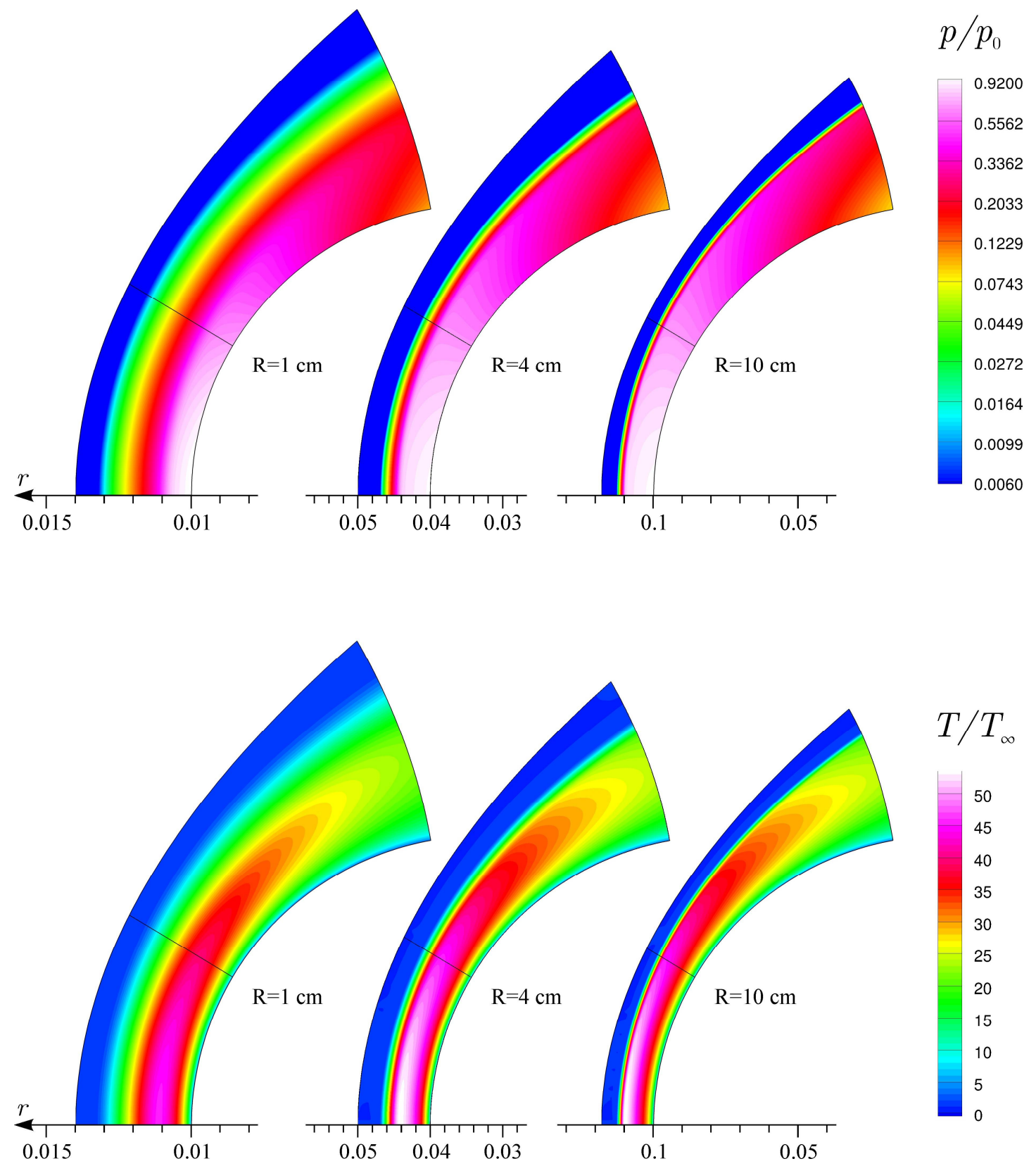

Рис. 1. Поля давления и температуры при различных радиусах носового затупления, $V_{\infty}=5000 \mathrm{M} / \mathrm{c}$

Количественно то же самое показывают распределения давления, температуры и скорости газа вдоль осевой линии на рис. 2 для скорости набегающего потока $5000 \mathrm{~m} / \mathrm{c}$ и $3500 \mathrm{M} / \mathrm{c}$. При $R=10 \mathrm{~cm}$ меньшая скорость набегающего потока приводит к увеличению толщины ударного слоя на $20 \%$, а при $R=1$ см толщина ударного слоя практически не изменяется. Хорошо заметно снижение температуры почти в 2 раза при меньшей скорости набегающего потока. 

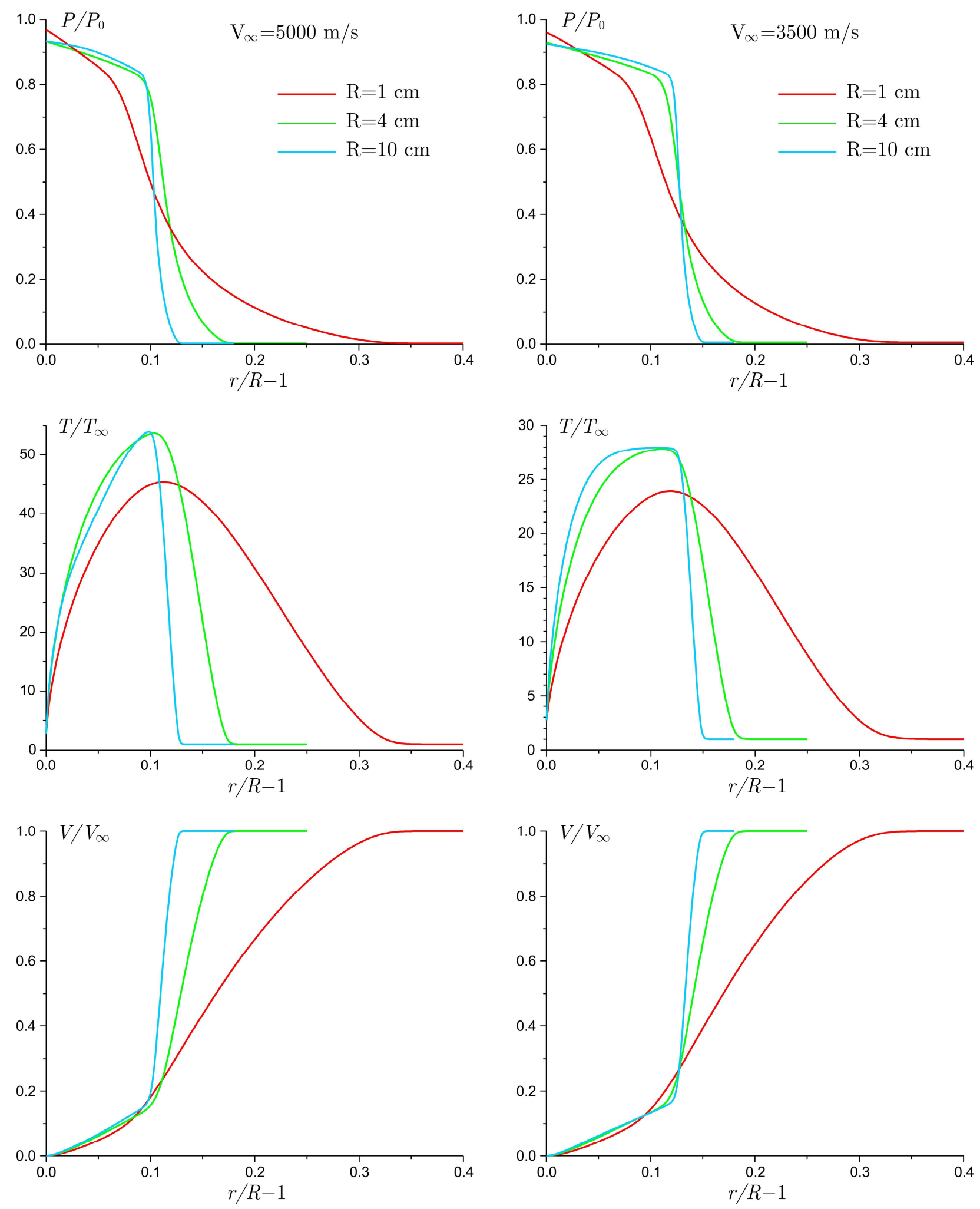

Рис. 2. Профили давления, температуры и скорости течения на оси симметрии при различных радиусах носового затупления. Левая колонка: $V_{\infty}=5000 \mathrm{~m} / \mathrm{c}$; правая колонка: $V_{\infty}=3500 \mathrm{M} / \mathrm{c}$

На рис. 3, 4 и 5 построены распределения массовых долей $\mathrm{O}, \mathrm{NO}$ и N соответственно вдоль осевой линии для обеих скоростей набегающего потока и трех радиусов затупления. Все графики показывают действие каталитических свойств поверхности: полностью каталитической (красные линии) и полностью некаталитической (синие линии). В первом случае все массовые доли на поверхности ожидаемо обращаются в ноль, во втором случае сохраняют конечные значения. 



Рис. 3. Массовые доли О на оси симметрии при различных радиусах носового затупления и различных условиях рекомбинации на поверхности. Левая колонка: $V_{\infty}=5000 \mathrm{~m} / \mathrm{c}$, правая колонка: $V_{\infty}=3500 \mathrm{M} / \mathrm{c}$

При этом у атомарного кислорода максимум всегда достигается на поверхности, а у азота максимум достигается в поле, а к поверхности уровень снижается. Это говорит о том, что атомарный азот более быстро реагирует на снижение температуры при подходе к по- 
верхности. Поведение азота сказывается и на NO: при $R=10$ см и скорости набегающего потока 5000 м/с уровень NO также снижается при подходе к поверхности.

NO mass fraction
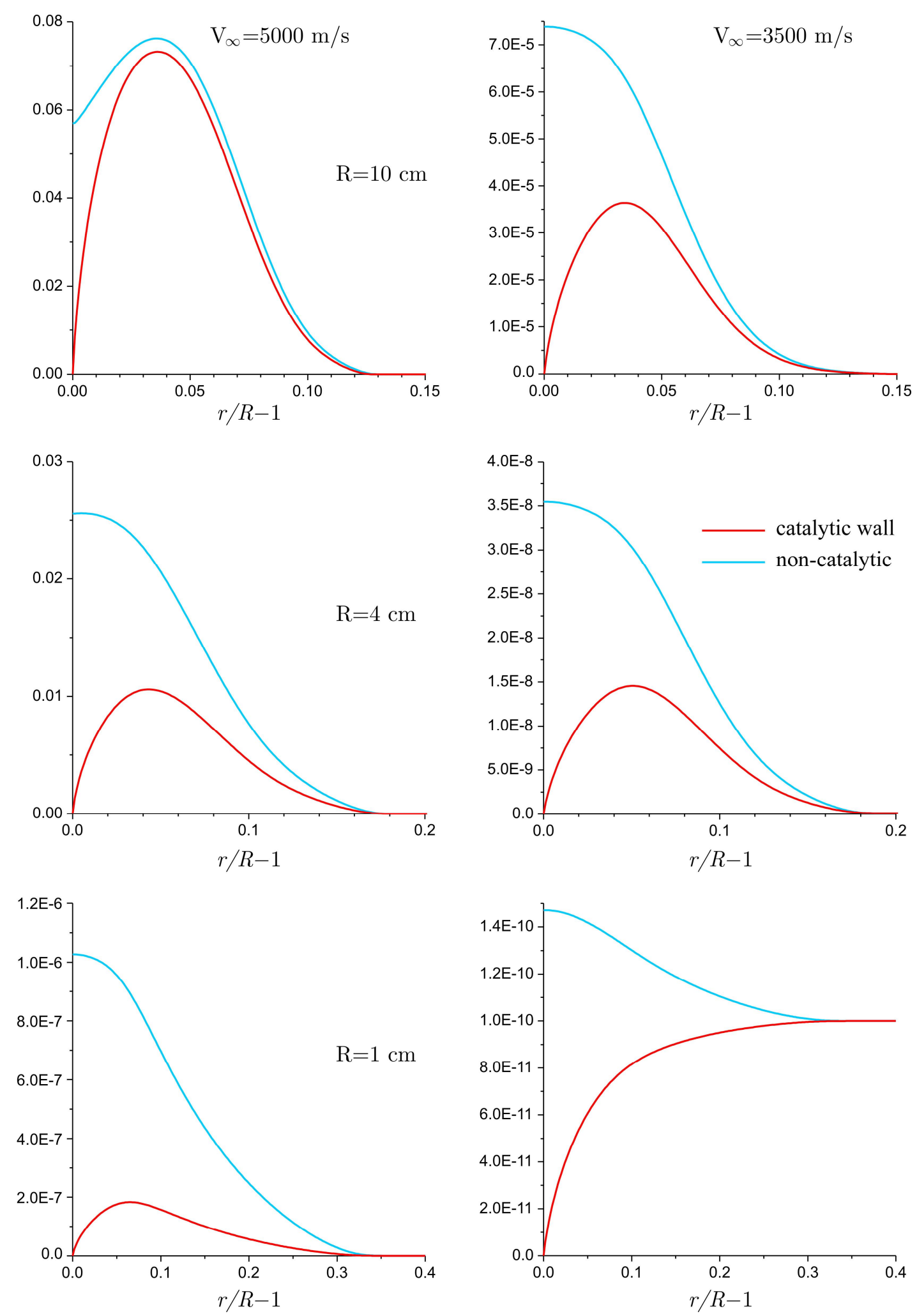

Рис. 4. Массовые доли NO на оси симметрии при различных радиусах носового затупления и различных условиях рекомбинации на поверхности. Левая колонка: $V_{\infty}=5000 \mathrm{~m} / \mathrm{c}$, правая колонка: $V_{\infty}=3500 \mathrm{M} / \mathrm{c}$ 

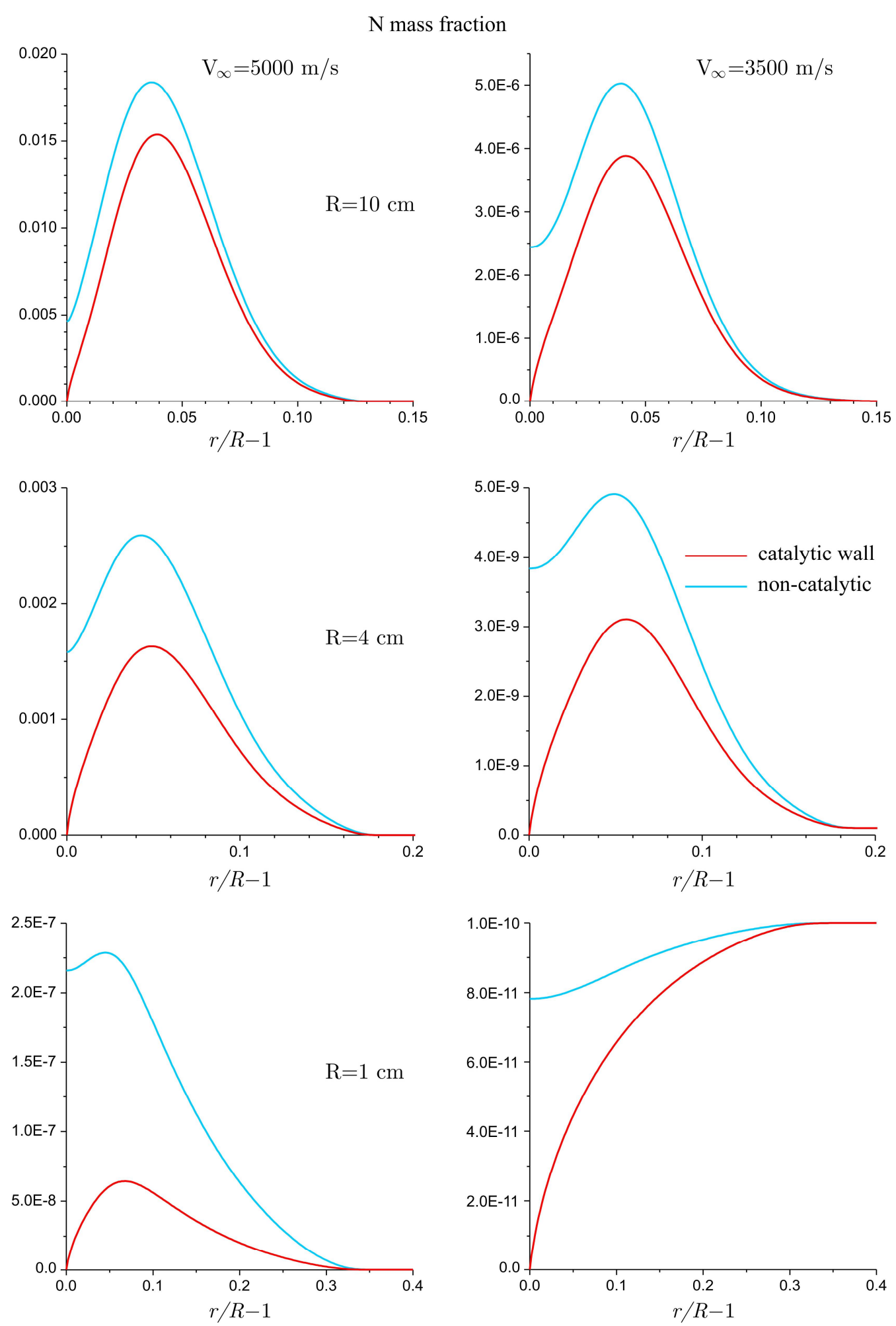

Рис. 5. Массовые доли $\mathrm{N}$ на оси симметрии при различных радиусах носового затупления и различных условиях рекомбинации на поверхности. Левая колонка: $V_{\infty}=5000 \mathrm{~m} / \mathrm{c}$, правая колонка: $V_{\infty}=3500 \mathrm{M} / \mathrm{c}$

Скорость набегающего потока сильно влияет на температуру в ударном слое и соответственно на скорость химических реакций. Поэтому в случае $3500 \mathrm{~m} / \mathrm{c}$ массовые доли компонент оказываются на несколько порядков ниже по сравнению со случаем 5000 м/c, а при $R=1$ см все массовые доли оказываются вблизи фонового уровня $10^{-10}$, который изначально задан в набегающем потоке в качестве граничного условия. В последнем случае химические реакции просто не успевают произойти. 
На рис. 6 приведены колебательные температуры $\mathrm{O}_{2}, \mathrm{NO}, \mathrm{N}_{2}$ на осевой линии в сравнении с поступательной температурой.
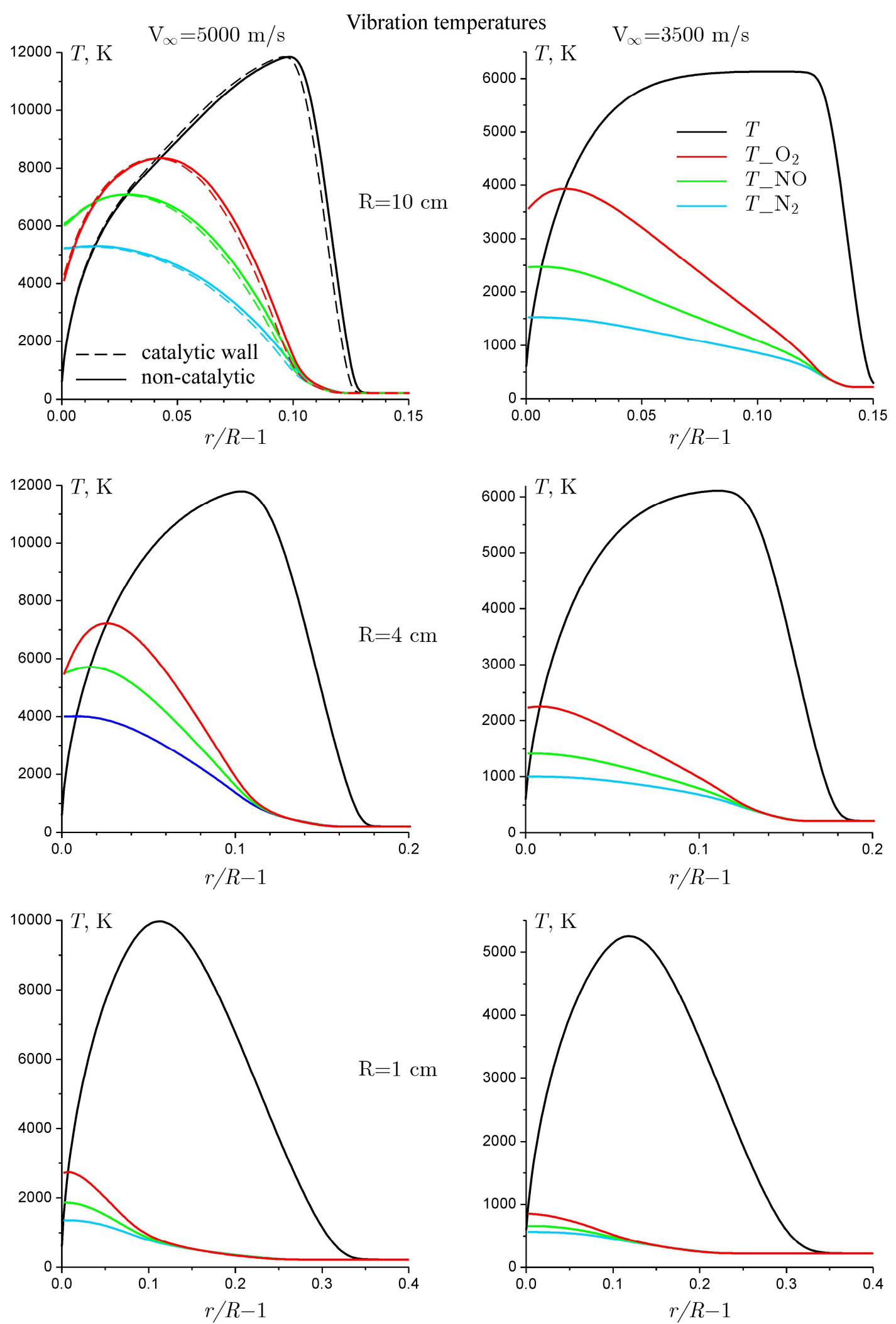

Рис. 6. Колебательные температуры двухатомных молекул в сравнении с поступательной температурой на оси симметрии при различных радиусах носового затупления. Левая колонка: $V_{\infty}=5000 \mathrm{~m} / \mathrm{c}$, правая колонка: $V_{\infty}=3500 \mathrm{~m} / \mathrm{c}$ 
Результаты говорят о значительной колебательной неравновесности. Все колебательные температуры оказываются ниже поступательной температуры, причем с уменьшением радиуса затупления отличие усиливается. Это естественно: чем меньше размер, тем меньше время пребывания частиц газа в поле течения, и релаксация не успевает произойти. Каталитические свойства поверхности не оказывают сколь-нибудь заметного влияния на колебательную неравновесность. Только при $R=10$ см и скорости набегающего потока $5000 \mathrm{~m} / \mathrm{c}$ видны небольшие отличия, показанные пунктиром. В остальных случаях отличия оказались меньше толщины линии. В таблицах 4 и 5 приведены значения теплового потока в критической точке в зависимости от скорости набегающего потока, радиуса затупления и каталитических свойств поверхности. Тепловой поток получается тем выше, чем больше скорость набегающего потока и меньше радиус затупления. Влияние каталитических свойств поверхности заметно только при максимальных значениях скорости и радиуса затупления, а с их уменьшением оно практически полностью исчезает. Для сравнения численных данных с результатами широко используемых инженерных формул для расчета тепловых потоков к критической точке сферического затупления (например, [17] с поправкой на разреженность [18]) такие результаты приведены в таблице 6.

\section{Таблица 4}

Тепловой поток к идеально некаталитической

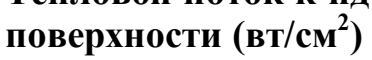

\begin{tabular}{|c|c|c|c|}
\hline & \multicolumn{3}{|c|}{$R, \mathrm{~cm}$} \\
\hline$V, \mathrm{M} / \mathrm{c}$ & 10 & 4 & 1 \\
\hline 5000 & 21.3 & 44.59 & 104.61 \\
\hline 3500 & 10.59 & 17.95 & 38.13 \\
\hline
\end{tabular}

\section{Таблица 5}

Тепловой поток к идеально каталитической поверхности (вт/см²)

\begin{tabular}{|c|c|c|c|}
\hline & \multicolumn{3}{|c|}{$R, \mathrm{~cm}$} \\
\hline$V, \mathrm{M} / \mathrm{c}$ & 10 & 4 & 1 \\
\hline 5000 & 30.82 & 48.43 & 104.62 \\
\hline 3500 & 10.62 & 17.95 & 38.13 \\
\hline
\end{tabular}

Таблица 6

Тепловой поток к идеально каталитической

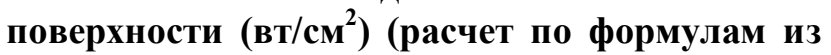
[17] и [18])

\begin{tabular}{|c|c|c|c|}
\hline & \multicolumn{3}{|c|}{$R, \mathrm{~cm}$} \\
\hline$V, \mathrm{M} / \mathrm{c}$ & 10 & 4 & 1 \\
\hline 5000 & 42.83 & 67.78 & 135.24 \\
\hline 3500 & 14.76 & 23.21 & 46.4 \\
\hline
\end{tabular}

Приведем кратко используемые в данной работе соотношения из работ Таубера [17] и Колодзея [18]. Здесь используется выражение для теплового потока (вт/м²) к критической точке в виде

$$
q_{\text {cont }}=1.83 \times 10^{-4}\left(\frac{\rho_{\infty}}{R}\right)^{0.5} V_{\infty}^{3}\left(1-\frac{h_{w}}{H_{s}}\right)
$$


Здесь $\rho_{\infty}$ - плотность набегающего потока; $R$ - радиус затупления; $V_{\infty}$ - скорость полета; $h_{w}$ - энтальпия на стенке; $H_{s}$ - полная энтальпия. Все в системе (СИ). Выбор соотношения из [17] для сравнения с результатами численного прогноза тепловых потоков определяется более современной работой и очень качественной и полной методикой изложения и анализом применения такого рода аналитических соотношений для различных видов гиперзвуковых течений.

Поправка Колодзея [18] на разреженность имеет вид полинома пятой степени, определяющего отношение тепловых потоков в разреженной (rare) и сплошной средах (cont). Ниже приведен сканированный из [18] рисунок зависимости $q_{\text {rare }} / q_{\text {cont }}(X)$.

$$
\begin{aligned}
& \frac{q_{\text {rare }}}{q_{\text {cont }}}=a_{0}+a_{1} X+a_{2} X^{2}+a_{3} X^{3}+a_{4} X^{4}+a_{5} X^{5} \\
& \begin{array}{ll}
a_{0} & -1.38151
\end{array} \\
& a_{1}+2.26375 \\
& a_{2} \quad-0.54005 \\
& a_{3}-0.02164 \\
& a_{4}+0.02016 \\
& a_{5} \quad-0.00170
\end{aligned}
$$

Здесь X - значения чисел Рейнольдса (Ret2), определенные по параметрам равновесного воздуха за прямой ударной волной. Зависимость поправки от Ret2 показана на графике из $[18] .{ }^{1}$



Сравнение тепловых потоков, полученных численной и инженерной методиками указывает на завышение конвективного теплового потока при использовании последней, что может быть объяснено разными используемыми подходами в учете физико- химической кинетики и газовой динамики (неравновесная кинетика и полные уравнения Навье-Стокса в расчетах) и обработка расчетов пограничного слоя с полностью равновесной кинетикой при выводе аналитических соотношений. Необходимо отметить, что условия обтекания те-

\footnotetext{
${ }^{1}$ Авторам данной работы не очень понятна методика получения этой зависимости и, тем более, привязка описания разреженного течения к числу Ret2, определяемому в равновесном и сплошносредном приближениях. Остается верить серьезному изданию и автору [18].
} 
ла с $R=1$ см соответствуют переходному между сплошносредным и разреженным режимами течения. В этом случае, необходим учет условий скольжения и скачка температуры на теле, как рекомендовано, например, в работе [19]. Поэтому, данные, приведенные в работе для обтекания тела с $R=1$ см носят количественно оценочный характер.

\section{5. Заключение}

На основе расчетов, выполненных с использованием программного комплекса HSflow, проведено исследование распределения параметров течения около сферического затупления тела радиуса $R=1,4,10$ см при скоростях набегающего потока $V_{\infty}=3.5 \mathrm{\kappa m} / \mathrm{c}$ и $5.0 \mathrm{kм} / \mathrm{c}$. Расчетные данные указывают на существенный уровень физико-химической неравновесности (колебательной, колебательно-диссоционной, диссоционной и за счет химических реакций) течения в вязком ударном слое в рассматриваемых условиях обтекания. Данные по величинам тепловых потоков к критической точке, полученные по программе HSFlow, ниже потоков, предсказываемых при помощи широко используемых на практике инженерных формул.

Работа выполнена при финансовой поддержке РФФИ Грант 18-08-00020

\section{Литература}

1. Gnoffo P.A., Gupta R.N., Shinn J.L. Conservation Equations and Physical Models for Hypersonic Air Flows in Thermal and Chemical Nonequilibrium. //NASA TP 2867, February 1989

2. Суржиков C.T. Расчет обтекания модели космического аппарата MSRO с использованием кодов NERAT-2D и NERAT -3D//Физико-химические процессы в газовой динамике. 2010. Том 10. www.chemphys.edu.ru/pdf/2010-01-12-003.pdf

3. Железнякова А.Л., Суржиков С.Т. Поле течения около космического аппарата FIRE II под углом атаки. //Физико-химические процессы в газовой динамике. 2010. Том 10. www.chemphys.edu.ru/pdf/2010-01-12-020.pdf

4. Millikan R.C., White D.R. Systematics of vibrational relaxation. //J. of Chemical Physics, V. 39, No 12, 1963, pp. 3209-3213.

5. Park C. Review of chemical-kinetic problems of future NASA missions. I. Earth entries.// J. of Thermophysics and Heat Transfer, V. 7, No 3, 1999, pp. 385-398.

6. Сергиевская А.Л., Ковач Э.А., Лосев С.А. Опыт информационно-математического моделирования в физико-химической кинетике. М.: Изд-во МГУ, 1995.

7. Егоров И.В., Новиков А.В. Прямое численное моделирование ламинарно-турбулентного обтекания плоской пластины при гиперзвуковых скоростях потока. //ЖВММФ, Т. 56, N 6, С. 1064 1081, 2016.

8. Годунов С.К. Конечно-разностный метод численного расчета разрывных решений уравнений газовой динамики // Матем. сборник. 1959. Т. 47. С. 271-291.

9. Годунов С.К., Забродин А.В., Прокопов Г.П. //Разностная схема для двумерных нестационарных задач газовой динамики и расчет обтекания с отошедшей ударной волной.// ЖВММФ, 1:6 (1961), C. 1020-1050

10. Roe P.L. Approximate Reimann solvers, parameter vectors, and difference schemes // J. Comp. Phys. 1981. V. 43. Pp. 357-372.

11. Колган В.П. Применение принципа минимальных значений производной к построению конечноразностных схем для расчета разрывных решений газовой динамики // Учёные записки ЦАГИ. 1972. Т. 3. № 6. С. 68-77.

12. Harten A. High resolution schemes for hyperbolic conservation laws // Journal of Computational Physics. 1983. V. 49. Pp. 357-372. 
13. Иванов М.Я., Крупа В.Г., Нигматуллин Р.З. Неявная схема С.К. Годунова повышенной точности для интегрирования уравнений Навье-Стокса // ЖВММФ. 1989. Т. 29. № 6. С. 888-901.

14. Каримов Т.Х. О некоторых итерационных методах решения нелинейных уравнений в гильбертовом пространстве // Докл. АН СССР. 1983. Т. 269. № 5. С. 1038-1046.

15. Saad Y., Shultz M.H. GMRes: a generalized minimal residual algorithm for solving nonsymmetric linear systems // SIAM J. Scient. and Statist. Comp. 1986. V. 7. № 3. Pp. 856-869.

16. Бабаев И.Ю., Башкин В.А., Егоров И.В. Численное решение уравнений Навье-Стокса с использованием итерационных методов вариационного типа // ЖВММФ. 1994. Т. 34 . № 11. C. $1693-1703$.

17. Tauber M. A Review of High-Speed Convective, Heat-Transfer Computation Methods, //NASA TP 2914, 1989.

18. Kolodziej P. Aerothermal Performance Constraints for Hypervelocity Small Radius Unswept Leading Edges and Nosetips, //NASA TM 112204, 1997.

19. Cuda V., Jr, Moss J.N. Direct Simulation of Hypersonic Flows Over Blunt Slender Bodies, //AIAA Paper -86-1348, 1986 